\section{Cureus}

\title{
Pregnancy or Uremia? - Case Report and Review of Conception, Pregnancy, and Complications in Peritoneal Dialysis Patients
}

\author{
Amarpreet Sandhu ${ }^{1}$, Anil Regmi ${ }^{2}$, David Buchwald ${ }^{3}$, Antonios H. Tzamaloukas ${ }^{4}$ \\ 1. Transplant Nephrology, California Pacific Medical Center, San Francisco 2. Division of Nephrology, \\ University of New Mexico School of Medicine, Albuquerque, New Mexico, USA 3. Division of Nephrology, \\ School of Medicine New Mexico, Albuquerque, New Mexico, USA 4. University of New Mexico School of \\ Medicine
}

$\square$ Corresponding author: Amarpreet Sandhu, sandhudoctor@gmail.com Disclosures can be found in Additional Information at the end of the article

\section{Abstract}

Pregnancy is infrequent in women undergoing renal replacement therapy (RRT) and may lead to serious maternal and fetal complications. Although rates of conception have increased for women on hemodialysis (HD), overall conception rates for women on renal RRT remain low. Incidence of pregnancy in peritoneal dialysis (PD) patients has been reported as $1.1 \%$ in a study that followed 1,699 patients for four years. The lower pregnancy rate in women on PD is due to mechanical factors on top of functional and physiological abnormalities. In the United States, the majority of the pregnant women undergoing PD are transitioned to HD to improve fetal outcomes. Due to negligible rates of conception and to similar symptoms, early pregnancy symptoms in patients with advanced chronic kidney disease (CKD) approaching RRT can be confused with uremic symptoms. Once pregnancy is established, multilevel changes need to be made in the management of women on PD. Coordinated care by nephrologists, neonatologists, obstetricians, and nutritionists can lead to better outcomes. We present a case of pregnancy in a woman with advanced CKD where PD was continued with good outcomes. The pregnancy was terminated by a full-term natural delivery. In addition, we review the diagnosis, management, outcomes, and complications of pregnancy in women on PD.

Categories: Internal Medicine, Medical Education, Miscellaneous

Keywords: pregnancy, delivery, peritoneal dialysis, dialysis, end stage renal disease, nephrology

Received 07/22/2014

Review began 07/22/2014

Review ended 08/31/2014

Published 09/17/2014

\section{Copyright 2014}

Sandhu et al. This is an open access article distributed under the terms of the Creative Commons Attribution License CC-BY 3.0., which permits unrestricted use, distribution, and reproduction in any medium, provided the original author and source are credited.

\section{Introduction}

Pregnancy is not only infrequent but also has a complicated course in women on dialysis. The first case of pregnancy in a woman on peritoneal dialysis (PD) was reported in 1980s. Since then, rates of conception in women on PD are increasing but still remain lower than the rates in women on hemodialysis (HD). However, there is no statistical difference in the live birth rates in PD versus HD patients. The diagnosis of pregnancy in women on dialysis is complicated by major difficulties. First, the pregnancy may be missed because many clinicians assume almost negligible rates of conception in dialysis patients. Second, certain clinical manifestations, e.g. nausea and vomiting, are common in uremia and early pregnancy. Third, the basic pregnancy tests are not reliable in women on dialysis because most of these markers are cleared by the kidneys and can give false positive results. Once pregnancy occurs in women on renal replacement therapy (RRT), multilevel coordinated care is needed for better outcomes. 
Management of pregnancy becomes even more challenging in women on PD, who are usually transitioned to HD. This report presents an illustrative case of pregnancy in a woman on PD with successful outcome followed by a review of the diagnostic and management strategies of pregnancy in this patient population.

\section{Case Presentation}

A 25-year-old woman with advanced CKD secondary to autosomal dominant polycystic kidney disease (ADPKD) was seen by the nephrologists with concerns for uremia. She had developed nausea, vomiting, anorexia, and loss of weight. Her medical history included hypertension, optic neuritis, pyelonephritis, asthma, left middle cranial fossa arachnoid cyst, and ADPKD. Her medications included valsartan, oxycodone, promethazine, and calcium with vitamin D.

At the time of the nephrology consultation, her serum creatinine was $2.7 \mathrm{mg} / \mathrm{dL}$ and estimated glomerular filtration rate (eGFR) was. $26 \mathrm{ml} / \mathrm{min} / 1.73 \mathrm{~m}^{2}$. Her weight was $46.2 \mathrm{~kg}$, height 172.7 $\mathrm{cm}$, and a calculated body mass index (BMI) was $15.5 \mathrm{~kg} / \mathrm{m}^{2}$. The low BMI prompted the measurement of creatinine clearance in a 24-hour urine specimen. Measured creatinine clearance was $15 \mathrm{~mL} / \mathrm{min}$, suggesting that CKD was far more advanced than the indication from eGFR. Considering the advanced CKD with low chances of conception in this population, no concern of pregnancy by the patient and unremarkable abdominal ultrasound findings, other than those of ADPKD, her symptoms were initially attributed to uremia.

Options of renal replacement therapy (RRT) were discussed with the patient, and she elected to have continuous ambulatory peritoneal dialysis (CAPD). Two weeks after placement of the PD catheter, she presented to the emergency department (ED) with persistent symptoms of nausea, vomiting, and now abdominal cramps. A computed tomography (CT) scan of abdomen and pelvis showed ascending colitis, possibly infectious or inflammatory, scattered free intraabdominal fluid, and an unremarkable uterus. Once again, given the low concern for pregnancy, $\beta$-HCG test was not done and she was discharged from the ED with the diagnosis of gastroenteritis.

She continued to have nausea and vomiting two months after initiation of PD despite adequate dialysis with weekly fractional urea clearance (Kt/V urea) of 3.2. As a part of further workup, $\beta$ HCG was tested and was positive. However, this test can be falsely positive in women with advanced CKD. Nevertheless, due to the suspicion of pregnancy, abdominal ultrasound was repeated and revealed 20 weeks of pregnancy. Her valsartan was discontinued and she was started on labetalol for hypertension management.

She was continued on CAPD and had coordinated care by a nephrologist, obstetrician, and nutritionist. PD prescriptions are shown in Table 1 . Dialysis adequacy was maintained with weekly Kt/V urea of $>3.0$ and blood urea nitrogen (BUN) of $<50 \mathrm{mg} / \mathrm{dl}$ throughout the pregnancy as shown in Figures 1-2. She was treated with increased doses of erythropoietin and iron for anemia, and calcitriol for secondary hyperparathyroidism. Hemoglobin, divalent cation, and bicarbonate levels in serum were maintained at the levels recommended by Kidney Disease Improving Global Outcomes - CKD guidelines (KDIGO-CKD). 


\section{Cureus}

\begin{tabular}{|l|l|l|l|l|l|}
\hline Time & $\begin{array}{l}\text { Dialysate (Dextrose } \\
\text { \%) }\end{array}$ & $\begin{array}{l}\text { Exchanges Per } \\
\text { Day }\end{array}$ & $\begin{array}{l}\text { Fill Volume } \\
\text { (I) }\end{array}$ & $\begin{array}{l}\text { Total Volume } \\
\text { (I) }\end{array}$ & $\begin{array}{l}\text { Dwell Time } \\
\text { (hours) }\end{array}$ \\
\hline $\begin{array}{l}\text { At the beginning } \\
24 \text { weeks of }\end{array}$ & $1.5 \%$ & 4 & 2 & 8 & 3 \\
\hline $\begin{array}{l}\text { gestation } \\
\text { Pre-delivery }\end{array}$ & $1.5 \%$ & 5 & 1.5 & 7.5 & 3 \\
\hline
\end{tabular}

TABLE 1: CAPD Prescriptions at Different Stages of Pregnancy

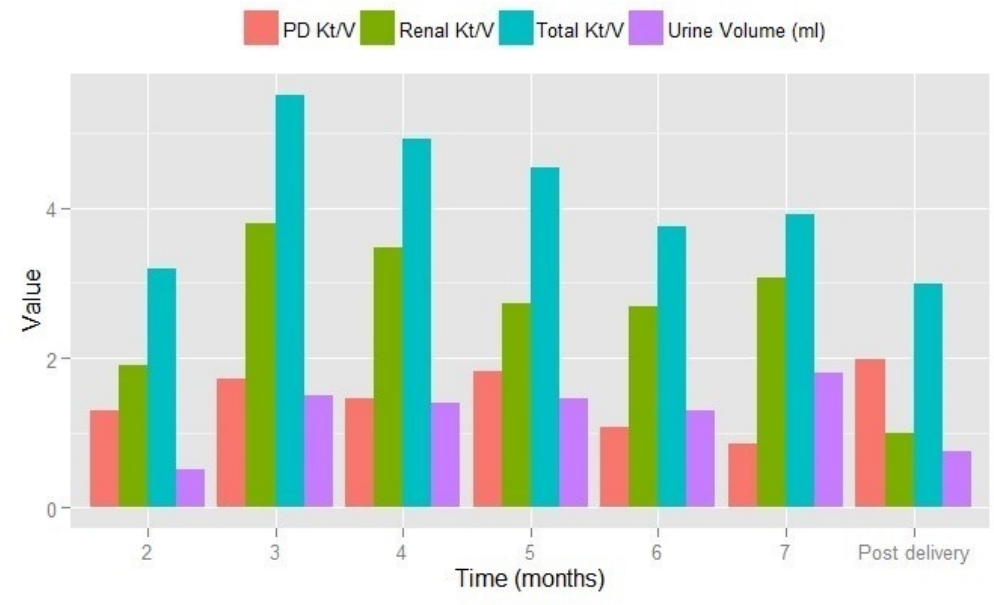

FIGURE 1: Dialysis adequacy was maintained with weekly Kt/V urea of $>3.0$ and blood urea nitrogen (BUN) of $<50 \mathrm{mg} / \mathrm{dl}$ throughout the pregnancy.

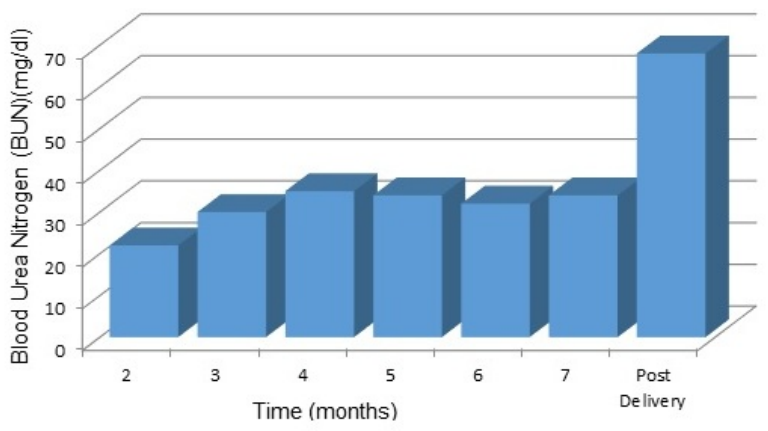

FIGURE 2: Dialysis adequacy was maintained with weekly Kt/V 


\section{urea of $>3.0$ and blood urea nitrogen (BUN) of $<50 \mathrm{mg} / \mathrm{dl}$ throughout the pregnancy.}

The pregnancy was uneventful, and labor was induced after she developed abdominal discomfort at 38 weeks and five days of gestation. The option of HD, discussed with the patient because she was not able to continue PD, was rejected by her. Instead, she chose induction of labor. She was continued on CAPD during labor with small frequent exchanges (Table 1). She delivered a healthy baby boy of $2.78 \mathrm{~kg}$, APGARS of $6 / 8$ over intact perineum on the third day of the hospitalization.

Regular CAPD prescription was resumed 12 hours after delivery. Both the mother and the baby were discharged home three days after delivery.

\section{Discussion}

Although pregnancy in end-stage renal disease (ESRD) patients is infrequent, recent data show that the conception rates are improving overtime. According to data from Registry of Pregnancy in Dialysis Patients (RPDP), conception rate in HD patients in a two-year period (1990-1992) was $1.5 \%$. This rate improved to $2.4 \%$ over a subsequent four-year period (1992-1995) [1-2]. Data from questionnaires in dialysis units of Saudi Arabia showed conception rates between 5.0\% and 7.9\% [3-4]. Recent data from Canada reported a conception rate of $15.9 \%$ in childbearing age women on intensive nocturnal hemodialysis [5]. Overall, conception in PD patients is extremely low. Based on data collection from 1699 childbearing age women on PD, conception rate is only $1.1 \%[1]$.

Most pregnancies reported to the RPDP have occurred in women during the first few years on dialysis; however, pregnancy has also been reported in women who were on dialysis for as long as 20 years. Even repeated pregnancies are not unknown. Out of reported 353 pregnant women by RPDP, eight became pregnant twice, eight became pregnant three times, and one became pregnant four times [1].

\section{Conception}

Menstrual irregularities, infertility, and sexual dysfunction are common in ESRD patients. These functional and physiological abnormalities worsen as renal disease progresses. Holley, et al. [6] described a progressive decline of physiological functions related to fertility: Menstrual cycle irregularities begin when glomerular filtration rate (GFR) falls below $15 \mathrm{ml} / \mathrm{min}$ and progress to amenorrhea at GFR values below $5 \mathrm{ml} / \mathrm{min}$.

Most studies of hormonal abnormalities in dialysis patients indicate that even women who menstruate have anovulatory cycles. Plasma levels of progesterone and estradiol are low, whereas hyperprolactinemia is seen in $70 \%-90 \%$ of the women. Luteinizing hormone (LH) levels are elevated, whereas follicle stimulating hormone (FSH) levels are comparable or slightly lower than in normal women during the follicular phase of menstrual cycle. Despite elevated levels of $\mathrm{LH}$, women on dialysis fail to have the luteal surge of $\mathrm{LH}$ that is directly related to ovulation [7-8 ]. Other factors that affect fertility in ESRD patients include subclinical hypothyroidism, medications, fatigue, anemia, and depression, which further results in lack of libido [9-10].

The lower conception rates in women on PD are in part due to mechanical factors on top of the functional and physiological irregularities. Hypertonic dextrose may damage the ovum and the 
volume of dialysate in the peritoneal space may interfere with ovum transfer within the fallopian tube. Recurrent peritonitis can also lead to fallopian tube obstruction [11].

Menstrual irregularities and amenorrhea make the diagnosis of pregnancy in ESRD women very challenging and delayed. Human chorionic gonadotropin (hCG) is partially cleared by the kidneys and false-positive serum pregnancy tests have been documented in this patient population. Since beta-hCG is inversely correlated with creatinine clearance and maternal serum pregnancy-associated plasma protein A (PAPP-A) is generally elevated in ESRD patients, these tests should be carefully interpreted as screening for pregnancy in the first trimester [12$13]$.

\section{Outcomes}

The first reported results of pregnancies in PD patients occurred in early 1988. In this series, Redrow, et al. [14] included eight pregnant women managed by PD, five of whom had successful deliveries. Theoretically, PD offers certain advantages for fetal survival, including constant biochemical and extracellular environment, higher hematocrit levels (this study was reported prior to the use of erythropoietin in large numbers of patients on hemodialysis), infrequent episodes of hypertension, and no requirements for systemic heparinization [14-15]. However, according to an early report by RPDP, there is no statistical difference in the live birth rate in PD (47.6\%) versus HD (46.4\%) patients [1]. In contrary, a single center study by Chow, et al. reported worse outcomes in PD patients [16]. Pregnancy in women undergoing RRT is associated with important maternal and fetal complications.

\section{Maternal risks and complications}

Maternal complications include premature birth, uncontrolled hypertension, miscarriage, placental detachment, anemia, infections, premature rupture of membranes, polyhydramnios, preeclampsia, eclampsia, hemorrhage, C-section, and even maternal death.

\section{Premature birth}

According to RPDP, 84\% of infants born to women on dialysis were born prematurely, $44 \%$ weighed less than 1500 grams, and 28\% were small for gestational age. Gestational age of survived infants born from women on PD ranged from 31 to 38 weeks [1].

\section{Hypertension}

Hypertension is very common in pregnant women on dialysis. About $80 \%$ of these women have blood pressure higher than 140/90 mm Hg and more than 50\% have blood pressure greater than 170/110 mm Hg. Theoretically, PD patients should have a lower risk of hypertension, given the continuous therapy and the hope of remaining euvolemic; however, small studies have shown no blood pressure difference between pregnant women on PD and those on hemodialysis [11].

Since hypertension in dialysis patients is often related to their volume status, it poses another challenge to determine dry weight in pregnant dialysis women (see below for details).

\section{Preeclampsia/eclampsia/HELLP Syndrome}

Preeclampsia is characterized by the development in pregnant women of high blood pressure and significant proteinuria after the $20^{\text {th }}$ week (i.e., late second or third trimester) of pregnancy. Preeclampsia left untreated can lead to eclampsia, an acute life-threatening condition with tonic-clonic seizures. HELLP syndrome is characterized by hematological alterations, including hemolysis and low blood platelet count, and by elevated levels of liver 
enzymes in the serum. HELLP syndrome can occur alone or as a complication in $10-20 \%$ of pregnant women with severe preeclampsia or eclampsia.

The absence of urine output in dialysis patients to determine proteinuria makes the diagnosis of preeclampsia difficult. In this patient population, the diagnosis relies on assessment of worsening blood pressure, alterations in placental Doppler blood flow, fetal growth retardation, and hematological abnormalities in case of HELLP syndrome [17].

\section{Urea clearance, polyhydramnios, and dialysis adequacy}

In pregnant women on dialysis, the BUN level is a most important marker of fetal outcome. Several retrospective studies and isolated clinical cases have reported increased fetal survival in women with BUN $<50 \mathrm{mg} / \mathrm{dl}$. Guidelines propose increasing the frequency of HD to four to six sessions per week to achieve at least 20 hours of dialysis per week and a pre-dialysis BUN level $1<50 \mathrm{mg} / \mathrm{dL}$ in pregnant women on HD [18-19]. Frequent HD sessions also help better control of hypertension, improvement in maternal nutrition, and prevention of polyhydramnios.

The incidence of polyhydramnios is approximately $30 \%-70 \%$ in pregnant women on dialysis. The amniotic fluid excess occurs because of increased production in fetal urine secondary to urea-induced osmotic diuresis. Increasing the dialysis dose as mentioned above in this patient population can help reduce this complication [20-21].

There is no commonly accepted target $\mathrm{Kt} / \mathrm{V}$ urea level for pregnant PD patients. However, Okundaye and Hou [22] reported a total (peritoneal, plus renal) Kt/V urea goal of $2.2-2.4$ weekly for better outcomes. These Kt/V urea values are difficult to achieve by CAPD alone. Residual renal function can be of great importance, as it was in our patient (Table 1). Intensive PD is very important in the third trimester when fetal urea production is increased to $540 \mathrm{mg}$ per day. Higher $\mathrm{Kt} / \mathrm{V}$ is achieved by increasing fill volume that might be difficult in pregnant patients due to discomfort, or by increasing the number of small volume exchanges, as we did in our patient.

\section{Electrolytes, minerals, vitamins, and acid-base abnormalities}

Several abnormalities occur because of intensive dialysis in this patient population. Hypokalemia worsens in intensively dialyzed pregnant PD patients and requires increased doses of potassium supplementation. Phosphate binders are usually discontinued in hemodialysis patients, and phosphate replacement may be needed in pregnant PD patients [22].

Water-soluble vitamins and minerals can be removed by intensive dialysis. Doubling of the dose of daily multivitamins, particularly folic acid, may be required. Pregnant women on PD do not have metabolic alkalosis that is usually seen immediately after HD and requires lower bicarbonate bath to maintain normal physiological bicarbonate goal of $25 \mathrm{meq} / \mathrm{L}$ [11].

\section{Anemia}

The etiology of anemia in pregnant women on dialysis is multifactorial. It includes iron and red blood cell loss from frequent hemodialysis, erythropoietin resistance probably from pregnancyinduced cytokines, and high demand of red blood cell production for fetal growth. Therefore, erythropoietin requirement increases by about $50 \%$, while the iron requirement is $30 \mathrm{mg} / \mathrm{day}$ [23].

\section{Weight gain and nutrition}


Determination of appropriate weight gain in pregnant dialysis patient is very difficult.

Approximately $9 \mathrm{~L}$ of total body water increase is expected in pregnancy. This increase leads to intravascular volume expansion due to vasodilatation, making fluid removal more difficult. In the first trimester, the mother should gain a minimum of 1-1.5 kilograms and 0.45-1 kilogram per week thereafter [19]. In general, estimated dry weight (EDW) should be increased to 400 grams per week after the first trimester to account for fetal weight of about 500 grams per week in the second and third trimester [21].

In pregnant women, nutritional assessment and counseling is important to ensure adequate protein and caloric intake. Providing adequate protein intake might be difficult in this patient population due to nausea, anorexia, and protein loss due to intensive dialysis. The pregnant dialysis women should take in 1 gram per kilogram per day protein, adding 20 grams per day for fetal growth [24]. Healthy maternal weight gain in a pregnant woman with a normal body mass index should be $11.5-15.9 \mathrm{~kg}$. For development of the fetal skeleton, the maternal diet should include an additional 30 grams of calcium supplementation [11].

\section{Peritonitis and bloody effluent}

The information on peritonitis in pregnant CAPD patients is limited. Out of three reported cases, labor occurred in two patients. One pregnancy resulted in stillbirth and the other pregnancy resulted in a premature baby who survived [22]. Five out of six pregnancies complicated by peritonitis reported by RPDP, resulted in surviving infants. One of these reported cases had a second trimester spontaneous abortion. Due to the connection between peritoneum and fallopian tubes, there is always risk of peritonitis from any pregnancyassociated infections. Penicillins and cephalosporins are the safest categories of antibiotics to use in pregnancy-associated peritonitis [22].

Bloody effluent is rarely a sign of a serious problem in non-pregnant PD patients. However, it is very concerning in pregnant PD patients. It may indicate placental abruption or impending abortion [14]. Patients with bloody effluent should be hospitalized for observation. Fetal ultrasound to look for placental separation should be performed.

\section{Anticoagulation}

Heparin does not cross the placenta and is not teratogenic. Therefore, it can be safely used on HD to prevent access clotting as well as maintenance of fibrin-free PD. Coumadin is contraindicated in these patients [25].

\section{Labor and delivery}

Intravenous magnesium must be administered with caution to pregnant women on PD to avoid toxicity and magnesium levels should be kept below 5-7 mg/dl [26].

Being on dialysis by itself does not warrant scheduling of C-section. However, if required for obstetric reasons, C-section can be done successfully in PD patients. The abdomen should be drained before surgery and surgery should be done extraperitoneally, if possible. Dialysis can be resumed with small volume exchanges 24 hours after the surgery. If PD fluid leakage occurs, the patients should be switched to HD for 2 weeks [11].

\section{Fetal risks and complications}

All infants born to this patient population should be observed in high risk setting, even if they appear normal and healthy. Most problems in the newborn are due to prematurity and growth retardation. Neonates are born with BUN and serum creatinine values close to those of their 
mother's. The high BUN values lead to osmotic diuresis and can cause significant volume contraction and electrolyte imbalance if losses are not replaced. Several retrospective studies and clinical cases have reported increased fetal survival in women with BUN $<50 \mathrm{mg} / \mathrm{dl}$. Conception before the start of RRT has been associated with higher incidence of live births (73.6\% vs. $40.2 \%$, respectively) and lower incidence of prematurity and low birth weight babies. Infants exposed to hypercalcemia during pregnancy are at increased risk of hypocalcemia and tetany after birth and need careful monitoring [11]. Breast milk will have high concentration of urea and in cases of breast-feeding, it can lead to osmotic diuresis in these infants [22].

\section{Conclusions}

Although infrequent, rates of pregnancy in ESRD women are increasing overtime. Besides the challenges of functional and physiological irregularities seen in hemodialysis patients, women on PD also face mechanical challenges. Pregnancy in ESRD patients poses diagnostic dilemmas. Evaluation of the possibility of pregnancy should be part of the care of women of childbearing age with advanced CKD and ESRD. Most of the patients who get pregnant while on PD are transitioned to daily HD during pregnancy with the goals of keeping their BUN $<50 \mathrm{mg} / \mathrm{dl}$. However, continuation of peritoneal dialysis can be an option in pregnant patients with preserved renal function. They require particular attention towards anemia management, blood pressure control, fluid status, dialysis prescription, nutrition, and fetal monitoring. Close collaboration between the patient, nephrologist, obstetrician, neonatologist, dialysis nurses, and nutritionist can lead to better outcomes.

\section{Additional Information}

\section{Disclosures}

Human subjects: Consent was obtained by all participants in this study. Conflicts of interest: In compliance with the ICMJE uniform disclosure form, all authors declare the following: Payment/services info: All authors have declared that no financial support was received from any organization for the submitted work. Financial relationships: All authors have declared that they have no financial relationships at present or within the previous three years with any organizations that might have an interest in the submitted work. Other relationships: All authors have declared that there are no other relationships or activities that could appear to have influenced the submitted work.

\section{Acknowledgements}

This case was presented at American Society of Nephrology Kidney week in November 2013.

\section{References}

1. Okundaye I, Abrinko P, Hou S: Registry of pregnancy in dialysis patients . Am J Kidney Dis. 1998, 31:766-73.

2. Hou SH: Frequency and outcome of pregnancy in women on dialysis . Am J Kidney Dis. 1994, 23:60-3.

3. Malik GH, Al-Harbi A, Al-Mohaya S, Dohaimi H, Kechrid M, Shetaia MS, Al-Hassan AO, Quiapos LS: Pregnancy in patients on dialysis--Experience at a referral center . J Assoc Physicians India. 2005, 53:937-41.

4. Bahloul H, Kammoun K, Kharrat M, Jarraya F, Charffedine K, Hamida MB, Hachicha J: Pregnancy in chronic hemodialysis women: Outcome of multicentric study . Saudi J Kidney Dis Transpl. 2003, 14:530-1.

5. Barua M, Hladunewich M, Keunen J, Pierratos A, McFarlane P, Sood M, Chan CT: Successful pregnancies on nocturnal home hemodialysis. Clin J Am Soc Nephrol. 2008, 3:392-6. 10.2215/CJN.04110907

6. Holley JL, Schmidt RJ, Bender FH, Dumler F, Schiff M: Gynecologic and reproductive issues in 
women on dialysis. Am J Kidney Dis. 1997, 29:685-90.

7. Lim VS, Henriquez C, Sievertsen G, Frohman LA: Ovarian function in chronic renal failure: Evidence suggesting hypothalamic anovulation. Ann Intern Med. 1980, 93:21-7.

8. Lim VS, Kathpalia SC, Frohman LA: Hyperprolactinemia and impaired pituitary response to suppression and stimulation in chronic renal failure: Reversal after transplantation. J Clin Endocrinol Metab. 1979, 48:101-7.

9. Bailie GR, Elder SJ, Mason NA, Asano Y, Cruz JM, Fukuhara S, Lopes AA, Mapes DL, Mendelssohn DC, Bommer J, Young EW: Sexual dysfunction in dialysis patients treated with antihypertensive or antidepressive medications: Results from the DOPPS. Nephrol Dial Transplant. 2007, 22:1163-70.

10. Steele TE, Wuerth D, Finkelstein S, Juergensen D, Juergensen P, Kliger AS, Finkelstein FO: Sexual experience of the chronic peritoneal dialysis patient. J Am Soc Nephrol. 1996, 7:11658.

11. Hou S: Conception and pregnancy in peritoneal dialysis patients . Perit Dial Int. 2001, 21:S290-4.

12. Buckner CL, Wilson L, Papadea CN: An unusual cause of elevated serum total beta hCG. Ann Clin Lab Sci. 2007, 37:186-91.

13. Shenhav S, Gemer O, Sherman DJ, Peled R, Segal S: Midtrimester triple-test levels in women with chronic hypertension and altered renal function. Prenat Diagn. 2003, 23:166-7.

14. Redrow M, Cherem L, Elliott J, et al.: Dialysis in the management of pregnant patients with renal insufficiency. Medicine (Baltimore). 1988, 67:199-208.

15. Lew SQ, Watson JA: Urea and creatinine generation and removal in a pregnant patient receiving peritoneal dialysis. Adv Perit Dial. 1992, 8:131-5.

16. Chou CY, Ting IW, Lin TH, Lee CN: Pregnancy in patients on chronic dialysis: A single center experience and combined analysis of reported results. Eur J Obstet Gynecol Reprod Biol. 2008, 136:165-70.

17. Hladunewich M, Hercz AE, Keunen J, Chan C, Pierratos A: Pregnancy in end stage renal disease. Semin Dial. 2011, 24:634-9. 10.1111/j.1525-139X.2011.00996.x

18. Asamiya Y, Otsubo S, Matsuda Y, Kimata N, Kikuchi K, Miwa N, Uchida K, Mineshima M, Mitani M, Ohta H, Nitta K, Akiba T: The importance of low blood urea nitrogen levels in pregnant patients undergoing hemodialysis to optimize birth weight and gestational age. Kidney Int. 2009, 75:1217-22. 10.1038/ki.2009.48

19. Giatras I, Levy DP, Malone FD, Carlson JA, Jungers P: Pregnancy during dialysis: Case report and management guidelines. Nephrol Dial Transplant. 1998, 13:3266-72.

20. Haase M, Morgera S, Bamberg C, Halle H, Martini S, Hocher B, Diekmann F, Dragun D, Peters $\mathrm{H}$, Neumayer HH, Budde K: A systematic approach to managing pregnant dialysis patients-the importance of an intensified haemodiafiltration protocol. Nephrol Dial Transplant. 2005, 20:2537-42.

21. Luders C, Castro MC, Titan SM, De Castro I, Elias RM, Abensur H, Romão JE Jr: Obstetric outcome in pregnant women on long-term dialysis: A case series. Am J Kidney Dis. 2010, 56:77-85. 10.1053/j.ajkd.2010.01.018

22. Okundaye I, Hou S: Management of pregnancy in women undergoing continuous ambulatory peritoneal dialysis. Adv Perit Dial. 1996, 12:151-5.

23. Barua M, Hladunewich M, Keunen J, Pierratos A, McFarlane P, Sood M, Chan CT: Successful pregnancies on nocturnal home hemodialysis. Clin J Am Soc Nephrol. 2008, 3:392-6. 10.2215/CJN.04110907

24. Ikizler TA, Flakoll PI, Parker RA, Hakim RM: Amino acid and albumin losses during hemodialysis. Kidney Int. 1994, 46:830-7.

25. Vázquez-Rodríguez JG: Hemodialysis and pregnancy: Technical aspects (article in Spanish) . Cir Cir. 2010, 78:99-102.

26. Keller F, Griesshammer M, Häussler U, Paulus W, Schwarz A: Pregnancy and renal failure: The case for application of dosage guidelines. Drugs. 2001, 61:1901-20. 